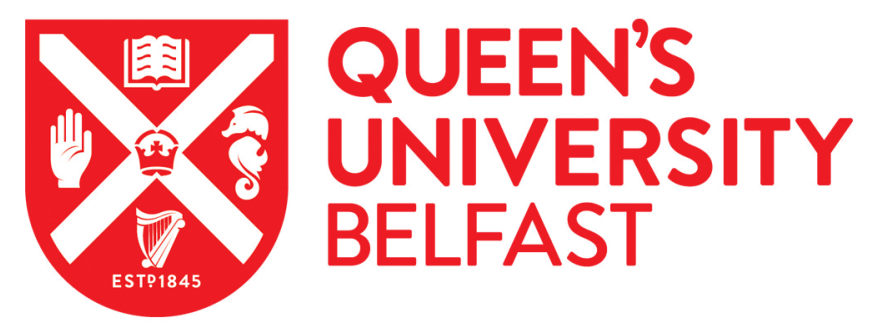

\title{
An Endogenously activated antiviral state restricts SARS-CoV-2 infection in differentiated primary airway epithelial cells
}

Broadbent, L., Bamford, C. G. G., Lopez Campos, G., Manzoor, S., Courtney, D., Ali, A., Touzelet, O., McCaughey, C., Mills, K., \& Power, U. F. (2021, Aug 18). An Endogenously activated antiviral state restricts SARS-CoV-2 infection in differentiated primary airway epithelial cells. https://doi.org/10.1101/2021.08.17.456707

Document Version:

Other version

Queen's University Belfast - Research Portal:

Link to publication record in Queen's University Belfast Research Portal

\section{Publisher rights}

Copyright 2021 the authors.

This is an open access preprint released under a Creative Commons Attribution-NonCommercial-NoDerivs License

(https://creativecommons.org/licenses/by-nc-nd/4.0/), which permits distribution and reproduction for non-commercial purposes, provided the author and source are cited.

\section{General rights}

Copyright for the publications made accessible via the Queen's University Belfast Research Portal is retained by the author(s) and / or other copyright owners and it is a condition of accessing these publications that users recognise and abide by the legal requirements associated with these rights.

Take down policy

The Research Portal is Queen's institutional repository that provides access to Queen's research output. Every effort has been made to ensure that content in the Research Portal does not infringe any person's rights, or applicable UK laws. If you discover content in the Research Portal that you believe breaches copyright or violates any law, please contact openaccess@qub.ac.uk. 


\section{An Endogenously activated antiviral state restricts SARS-CoV-2 infection in differentiated primary airway epithelial cells}

4 Lindsay Broadbent ${ }^{1^{*}}$, Connor G.G. Bamford ${ }^{1}$, Guillermo Lopez Campos ${ }^{1}$, Sheerien

5 Manzoor $^{1}$, David Courtney ${ }^{1}$, Ahlam Ali ${ }^{1,2}$, Olivier Touzelet ${ }^{1}$, Conall McCaughey ${ }^{3}$, Ken

1. Wellcome-Wolfson Institute for Experimental Medicine, Queens University Belfast, Belfast, Northern Ireland, United Kingdom.

2. Patrick G Johnston Centre for Cancer Research, Queen's University Belfast, Belfast, UK.

3. Regional Virus Laboratory, Belfast Health and Social Care Trust, Belfast, Northern Ireland. 
23 Severe acute respiratory syndrome coronavirus 2 (SARS-CoV-2), the cause of the

24 coronavirus disease-19 (COVID-19) pandemic, was identified in late 2019 and went on

25 to cause over 3.3 million deaths in 15 months. To date, targeted antiviral interventions

26 against COVID-19 are limited. The spectrum of SARS-CoV-2 infection ranges from

27 asymptomatic to fatal disease. However, the reasons for varying outcomes to SARS-CoV-

282 infection are yet to be elucidated. Here we show that an endogenously activated

29 interferon lambda (IFN $\lambda$ ) pathway leads to resistance against SARS-CoV-2 infection.

30 Using a well-differentiated primary nasal epithelial cell (WD-PNEC) model from multiple

31 adult donors, we discovered that susceptibility to SARS-CoV-2 infection, but not

32 respiratory syncytial virus (RSV) infection, varied. One of four donors was resistant to

33 SARS-CoV-2 infection. High baseline IFN $\lambda$ expression levels and associated interferon

34 stimulated genes correlated with resistance to SARS-CoV-2 infection. Inhibition of the

35 JAK/STAT pathway in WD-PNECs with high endogenous IFN $\lambda$ secretion resulted in

36 higher SARS-CoV-2 titres. Conversely, prophylactic IFN $\lambda$ treatment of WD-PNECs

37 susceptible to infection resulted in reduced viral titres. An endogenously activated IFN $\lambda$

38 response, possibly due to genetic differences, may be one explanation for the differences

39 in susceptibility to SARS-CoV-2 infection in humans. Importantly, our work supports the

40 continued exploration of IFN $\lambda$ as a potential pharmaceutical against SARS-CoV-2

41 infection. 
In late 2019 a novel coronavirus, severe acute respiratory syndrome coronavirus 2 (SARS-CoV-2), emerged in Hubei province in China ${ }^{1,2}$. This virus is the causative agent of the coronavirus disease-19 (COVID-19) pandemic, which has resulted in over 4.2 million deaths worldwide (as of August 2021). SARS-CoV-2 can infect people of any age. However, severe disease disproportionately affects older individuals or those with underlying health conditions. Clinical manifestations of SARS-CoV-2 infection range from asymptomatic or very mild symptoms to severe illness requiring hospitalization and death. COVID-19 disease is primarily a lung disease, typically manifesting itself as acute respiratory distress syndrome in severe cases, although extra-pulmonary complications, including cardiac, vascular, kidney and neurological pathologies, are also evident ${ }^{3}$. Furthermore, coagulopathies are commonly reported in COVID-19 disease patients. Reasons for the extreme variation in disease outcomes remain to be elucidated.

The upper respiratory tract is the primary site of SARS-CoV-2 infection. Infection can progress to the lower airways resulting in airway inflammation and potentially fatal pneumonia ${ }^{1}$. The major receptor for the viral spike protein (S), human angiotensinconverting enzyme 2 (ACE2), is expressed on airway epithelial cells but is also found on other tissue, including endothelial cells and smooth muscle cells ${ }^{4}$. Entry of the virus

62 requires activation of the $S$ glycoprotein by host proteases, such as furin and

63 transmembrane serine protease 2 (TMPRSS2) ${ }^{5}$. SARS-CoV-2 is cytopathic to airway

64 epithelial cells and alveolar cells. However, immune responses induced following infection 65 are also thought to drive pathogenesis and disease severity in COVID-19. Chemokines 66 and cytokines are released from infected tissues promoting leukocyte recruitment to the 67 lungs, resulting in substantial inflammation in severe cases. Hallmarks of severe COVID6819 disease include high serum levels of IL-6, TNF- $\alpha$, IP-10/CXCL10 and MCP-1, among 69 others ${ }^{6,7}$.

70 Dependent on viral, host and environmental factors, the IFN response to infection, and 71 subsequent inflammation, can be either beneficial or deleterious to the individual. Type I 72 and $\lambda$ (type III) interferons (IFNs) are produced in response to viral infection. IFN- 
73 mediated activation of the JAK-STAT pathway is responsible for the dramatic alteration

74 in the cellular transcriptome and the activation of interferon-stimulated genes (ISGs), 75 many of which have antiviral activities ${ }^{8}$. Patients with COVID-19 have low serum levels of

76 type I and III IFNs but high levels of pro-inflammatory chemokines and cytokines ${ }^{9}$.

77 However, it has been observed that higher serum IFN correlates positively with viral load

78 and disease severity ${ }^{10}$. It is hypothesized that dysregulated, reduced or delayed IFN

79 responses, in combination with high cytokine levels, results in more severe COVID-19

80 disease ${ }^{11}$.

81 Unlike type I IFN receptors, the IFN $\lambda$ receptor, IFNLR1, is not ubiquitously expressed and

82 is present predominantly on epithelial cells at barrier tissues like the lungs and 83 gastrointestinal tract ${ }^{12}$. As such, type III IFNs are considered to produce a more localised 84 response to infection in comparison to the systemic inflammatory response often induced 85 by type I IFN ${ }^{13}$. SARS-CoV-2 has been shown to induce an IFN response in epithelial 86 cells, the timing of which is delayed with regard to peak viral replication. Pre-treatment of 87 cells with IFNs is effective at blocking SARS-CoV-2 infection, and several directly antiSARS-CoV-2 ISGs have been discovered, such as Ly6, OAS1 and IFITMs ${ }^{14-16 .}$

As the primary target of SARS-CoV-2 infection is the airway epithelium, models that

90 authentically recreate the physiology and morphology of human airway epithelium in vivo

91 will undoubtedly be important tools to address fundamental questions about

92 human/SARS-CoV-2 interactions and subsequent innate immune responses. Therefore,

93 in this study we report differential susceptibility to SARS-CoV-2 infection in well-

94 differentiated primary nasal epithelial cell (WD-PNEC) cultures derived from adult donors.

95 We obtained nasal brushings from 4 healthy donors with no previous history of SARS-

96 CoV-2 infection. Interestingly, we found dramatically different susceptibility to SARS-CoV-

972 infection, with 1 of the 4 donors being resistant. The resistance was likely mediated by

98 high endogenous IFNL expression and associated ISG responses. These data may

99 provide an explanation as to why some individuals experience asymptomatic infection

100 following exposure to SARS-CoV-2. 


\section{Resistance to SARS-CoV-2 infection in WD-PNECs}

104 WD-PNEC cultures derived from 4 healthy adult donors demonstrated different 105 susceptibility to SARS-CoV-2 infection and viral growth kinetics (Figure 1A). Following 106 inoculation apical washes were harvested every $24 \mathrm{~h}$ post infection and titrated by plaque 107 assay on Vero cells. WD-PNECs from 3 donors demonstrated similar SARS-CoV-2 108 growth kinetics with peak viral titres of $>10^{6} \mathrm{PFU} / \mathrm{mL}$ at $48 \mathrm{hpi}$ followed by a plateau or 109 decrease at 72 hpi. Surprisingly, infection of donor 122 did not result in productive 110 infection of SARS-CoV-2, with virus titres continually diminishing relative to titres following 111 inoculation. Immunofluorescence reinforced these results, with high levels of SARS-CoV$1122 \mathrm{~N}$ expression in WD-PNECs from donors 311, 43 and 32, but with very few infected 113 cells evident in those from donor 122 (Figure 1C). Consistent with other data, SARS-CoV1142 infection was restricted to the apical surface of the cultures ${ }^{17}$. To determine if resistance 115 to infection was specific to SARS-CoV-2, we infected cultures derived from the same 116 donors with respiratory syncytial virus (RSV), a RNA virus that primarily infects ciliated 117 airway epithelium ${ }^{18}$. There was no evidence of restrictions in RSV growth kinetics in any 118 donor. (Figure 1B).

\section{Pre-activation of IFN pathway in resistant WD-PNECs}

120 The timing and magnitude of IFN responses to SARS-CoV-2 infection is thought to play

121 a role in disease severity. Transcriptomic analysis of RNA extracted at 48 and 96 hpi 122 revealed that there was upregulation of genes associated with the IFN $\lambda$ pathway in the 123 mock-infected cultures of donor 122. Following SARS-CoV-2 infection the gene 124 transcription signature of donor 122 did not significantly change (Figure 2A). Similar, or 125 greater, levels of expression of ACE2 and TMPRSS2 were observed in this donor, 126 confirming that resistance to infection was not due to absence or low expression of the 127 attachment and entry factors needed for SARS-CoV-2 infection. This was confirmed by 128 immunofluorescence (data not shown). The three donors susceptible to infection had 129 similar patterns of gene expression, including an increase in expression of IFN $\lambda$ - 
130 associated genes following SARS-CoV-2 infection (Figure 2A). To confirm these results,

131 IFN $\lambda 1$ concentrations in basolateral medium from SARS-CoV-2- or mock-infected WD-

132 PNECs ( $\mathrm{n}=4$ donors) were determined at 48 and $96 \mathrm{hpi}$. Increased IFN $\lambda 1$ secretion was

133 evident following infection at 96 hpi in cultures from donors 43 and 32, but not 311 . In

134 contrast, donor 122 cultures, which were not permissive to SARS-CoV-2 infection, had

135 high levels of IFN $\lambda 1$ (> $500 \mathrm{pg} / \mathrm{mL})$ in the basolateral medium from mock-infected cultures

136 at 48 and $96 \mathrm{hpi}$ (Figure 2B). Transcriptomic analysis of the IFIH1-mediated interferon

137 induction and toll-like receptor (TLR) signaling pathways revealed that donor 122 had a

138 unique gene transcription signature compared to mock or infected cultures from the other

139 donors (Supplementary Figure 1).

140 Pre-activation of IFN pathway and SARS-CoV-2 resistance phenotype are not 141 transient.

142 To determine if the high baseline level of IFN $\lambda 1$ in donor 122 cultures was an artifact of 143 culture or the time at which the nasal brush was obtained, a second brush was taken 4 144 months after the first and cultured in the same manner. When fully differentiated the 145 second cultures were infected with SARS-CoV-2, with similar results (Figure 3A). WD146 PNECs cultured from the second nasal brush of donor 122 also had high endogenous 147 levels of IFN $\lambda(\sim 400 \mathrm{pg} / \mathrm{mL})$, albeit reduced compared to those evident in the cultures 148 from the first brush. In contrast, IFN $\lambda$ levels from mock-infected cultures from 6 other 149 donors demonstrated background levels of secretion (Figure 3B).

\section{IFN signaling restricts SARS-CoV-2 infection}

151 SARS-CoV-2 is sensitive to exogenous IFN treatment ${ }^{19}$. We hypothesised that the high 152 baseline level of IFN $\lambda$ in basolateral medium of donor 122-derived WD-PNECs limited the 153 spread of SARS-CoV-2 within the culture and prevented productive infection. Therefore, 154 we blocked the major signalling pathway of IFN $\lambda$ with the JAK1/2 inhibitor ruxolitinib prior 155 to infection. WD-PNECs derived from a donor with low IFN $\lambda$ secretion in response to 156 SARS-CoV-2 infection (donor 311) was used as a control. Ruxolitinib treatment resulted 157 in a massive increase in SARS-CoV-2 titres in apical washes from cultures derived from 158 donor 122 (>3 $\log _{10} \mathrm{PFU} / \mathrm{mL}$ ), reaching a peak titre of $7.6 \log _{10} \mathrm{PFU} / \mathrm{mL}$ at $72 \mathrm{hpi}$ (Fig. 
$1594 \mathrm{~A})$. As expected, there was no significant effect of ruxolitinib treatment on donor 311 , 160 which also reached peak viral titres at $72 \mathrm{hpi}$. Viral titres at $72 \mathrm{hpi}$ were comparable for

161 untreated donor 311 cultures and ruxilitinib-treated donor 122 cultures, 7.78 and $7.6 \log _{10}$ $162 \mathrm{PFU} / \mathrm{mL}$, respectively.

163 To demonstrate that IFN $\lambda$ was the underlying cause of resistance to SARS-CoV-2 164 infection we replicated these conditions in the SARS-CoV-2 permissive donor 43. WD165 PNECs were pre-treated with IFN $\lambda$ in the basolateral medium for $24 \mathrm{~h}$ prior to infection 166 (Figure 4B). Treatment with both 1 and $100 \mathrm{ng} / \mathrm{mL}$ IFN $\lambda$ greatly reduced viral titres at 48 167 hpi by $>2 \log _{10} \mathrm{PFU} / \mathrm{mL}$, resulting in titres similar to the input inoculum (4.18 $\log _{10}$ $168 \mathrm{PFU} / \mathrm{mL})$.

169 Single nucleotide polymorphisms (SNP) analysis revealed differences in 170 pathogen sensing and interferon induction pathways unique to the resistant 171 donor

172 Unbiased SNP analysis from our RNAseq data demonstrated clear differences between 173 donors, with unique variants in several genes in the SARS-CoV-2-resistant donor 174 compared to the permissive donors. Focusing our SNP analysis to selected genes 175 associated with antiviral responses, we identified 388 SNPs unique to donor 122 and a 176 further 33 present in donors 311, 43 and 32 but not detected in donor 122 (Figure 5A).

177 Further conditional filtering for quality and existing SNP (according to dbSNP, NIH)

178 identified 10 SNPs potentially involved in the high endogenous IFN secretion and 179 resistance to SARs-CoV-2 demonstrated by WD-PNECs derived from donor 122 180 (Figure 5B). Interestingly, SNPs unique to donor 122 were found in ADAR (rs3738032), 181 MAVS (rs2089960995), TLR1 (rs4833095), TLR2 (rs1816702; rs3804099) and TLR3 182 (rs3775296; rs3775291). SNPs present in all donors except 122 were found in ADAR 183 (rs1127313; rs1127326) and IFIH1 (rs1990760). Together these results show that 184 resistant versus permissive donors have unique constellations of SNPs that may cause 185 the molecular mechanisms of susceptibility or resistance to SARS-CoV-2 infection. 


\section{Discussion}

188 The WD-PNEC model presents an ideal opportunity to study SARS-CoV-2 infection in a 189 clinically relevant system. The upper respiratory epithelium is thought to be the initial site

190 of infection for SARS-CoV-220. As such, a comprehensive understanding of SARS-CoV-

1912 infection of nasal epithelium is critical to understanding the early events of the infection

192 process, as they are likely influential in subsequent pathogenesis. Why some patients

193 with COVID-19 experience no or mild symptoms consistent with an upper respiratory tract

194 infection, while others experience much more severe symptoms often resulting in acute 195 respiratory distress syndrome (ARDS) remains to be determined. Consistent with earlier

196 reports, we demonstrated that differentiated nasal epithelial cells can be productively 197 infected with SARS-CoV-2 and that infection is primarily restricted to the apical surface.

198 Surprisingly, however, we provide robust evidence of differential susceptibility of airway 199 epithelium to SARS-CoV-2 infection, with airway epithelium from some individuals being 200 highly susceptible and others resistant. Our data suggest that resistance is likely 201 mediated in part by type III IFNs. Thus, we provide a potential explanation as to why some 202 individuals may be able to control initial SARS-CoV-2 infection.

203 The household secondary attack rate for SARS-CoV-2 is estimated to be $16.6 \%$, higher 204 than that of SARS-CoV $(7.5 \%)$ and MERS $(4.7 \%)^{21}$. While this demonstrates that 205 household contacts play a significant role in onward transmission of the virus it also 206 highlights that not everyone, despite close contact with an infected individual, will be 207 infected with SARS-CoV-2. Studies on pre-existing immunity to SARS-CoV-2 infections 208 have focused on aspects of the adaptive immune system and have demonstrated reactive 209 T cells in SARS-CoV-2-naive patients being important, possibly due to prior infections 210 with an endemic coronavirus ${ }^{22}$. However, differences in susceptibility to infection due to 211 innate immunity have not been reported previously. Interestingly, a recent pre-print 212 demonstrates a link between inter-individual differences in the expression of anti-SARS213 CoV-2 alleles of OAS1 in determine IFN-mediated cellular resistance to infection and 214 subsequent disease ${ }^{23}$. However, in our system, we observed enhanced endogenous 215 secretion of IFN and downstream antiviral ISGs. We propose that resistance to SARS216 CoV-2 infection is, in part, due to endogenous secretion and signaling of relatively high 
217 levels type III IFN in the mucosae independent of SARS-CoV-2 infection. This fits with 218 recent work showing high sensitivity of SARS-CoV-2 to prophylactic IFN treatment. 219 SARS-CoV-2 sensitivity to type I and III IFN treatment was previously reported. However, 220 contrary to our findings, these authors did not observe an interferon response to SARS221 CoV-2 infection of primary human airway epithelial cells ${ }^{17}$. A study by Dee et al. 222 demonstrated that rhinovirus infection induced an IFN response that blocked subsequent 223 SARS-CoV-2 infection. They proposed that given the prevalence of rhinovirus infections 224 within the population, it could impact the number of COVID-19 cases $^{24}$. In our model, the 225 underlying cause of such IFN secretion remains to be elucidated. Although this 226 phenomenon has only been observed in nasal cells from one donor it provides an exciting 227 precedent to explain resistance to SARS-CoV-2 infection. A large-scale study to 228 determine the frequency of endogenously-activated IFN responses may help to further 229 elucidate and explain differential susceptibility to COVID-19 disease in humans.

230 WD-PNECs that were resistant to SARS-CoV-2 infection were derived from a donor with 231 no known history of SARS-CoV-2 infection, was unvaccinated against COVID-19 at the 232 time of both nasal brush samplings, and was otherwise in good health. We cannot 233 discount the possibility that the donor had a prior bacterial or viral infection that, while still 234 allowing the epithelial cells to grow and differentiate, resulted in IFN $\lambda$ production in 235 uninfected WD-PNEC cultures. Furthermore, cultures were grown and differentiated in 236 the presence of antibiotics. Similarly, epigenetic changes are known to occur following 237 viral infection. Of particular note is influenza-induced epigenetic methylation of H3K79, 238 which may play an important role in interferon signaling ${ }^{25}$. That the WD-PNECs cultured 239 from the second nasal brushing 4 months after the initial sample were also resistant to 240 SARS-CoV-2 infection, suggests that this high endogenous level of IFN $\lambda$ was not a 241 transient response, consistent with a genetic or epigenetic mechanism. Indeed, SNP 242 analysis highlighted differences in IFN pathway genes in the donor that was resistant to 243 SARS-CoV-2 infection compared to those who were permissive. The presence of SNPS 244 in Toll-like receptors (TLRs) is known to affect susceptibility to disease ${ }^{26}$. Here we 245 identified several SNPs unique to donor 122 in TLR1, 2 and 3. Of particular note is a 246 common variant (minor allele frequency of 0.3 globally) in TLR3 (rs3775291; C>T) which 247 may confer resistance to HIV infection ${ }^{27}$. The mechanism of HIV resistance is still 
248 unknown. However, heterozygous individuals have higher cytokine mRNA levels

249 compared to $\mathrm{C}$ homozygotes which could restrict viral infection ${ }^{28}$. This phenomenon

250 appears to be virus specific as the same mutation actually confers susceptibility to

251 enteroviral infection ${ }^{29}$.

252 Treatment of human CoV infection with recombinant interferon is not a new concept. Type

253 I IFNs were tested against both SARS-CoV and MERS-CoV with promising pre-clinical

254 results but efficacy was less convincing in clinical trials ${ }^{30,31}$. Early clinical trials assessing

255 the efficacy of type I IFN treatment for COVID-19, alone or in combination with other

256 pharmaceuticals, have initially proved promising but further data are needed to draw

257 definitive conclusions. Similarities between type I and III IFN-induced signaling and

258 induction of antiviral and inflammatory responses are well known. However, type III IFN

259 effects are greatest in mucosal tissues, coincident with the tissue location of its receptor

260 on epithelial cells. Induction of type III IFNs results in prolonged expression of ISGs ${ }^{32,33 .}$

261 Type III IFNs have been trialed for treatment of hepatitis C virus (HCV), and hepatitis delta

262 virus (HDV) and, more recently, SARS-CoV-2, with positive results ${ }^{34-36}$. Type III IFNs,

263 with tissue-restricted receptor expression, are potentially a much more attractive option

264 for treatment of viral infection than the more inflammatory type I IFNs with ubiquitous

265 receptor expression. Our data supports further investigation into IFN $\lambda$, directed towards

266 the luminal surface of the airways, to reduce the incidence of severe COVID-19. Here we

267 have identified donor-specific resistance to SARS-CoV-2 infection in WD-PNECs

268 concurrent with high endogenous IFN $\lambda$, likely due to genetic differences. These findings

269 point to a previously overlooked mechanism of resistance that could be operating on a

270 global population level. 
274 The SARS-CoV-2 clinical isolate BT20.1 was isolated from a patient admitted to Royal

275 Victoria Hospital, Belfast in June 2020. Characterisation of this strain has previously been

276 described ${ }^{37}$. The virus was passaged 4 times on Vero cells, including isolation. The titres

277 of the virus stocks and experimental samples were determined by plaque assay. In brief,

278 Vero cells were seeded at $5 \times 10^{4}$ cells/well in a 24 -well cell culture plate. Twenty-four

279 hours later a 1:10 serial dilution of the virus stock or sample was prepared and inoculated

280 onto the Vero cells. Following $1 \mathrm{~h}$ incubation at $37^{\circ} \mathrm{C}$ a semi-solid agarose overlay was

281 added. Vero cells were further incubated for 3 days then fixed with paraformaldehyde

282 (final concentration $4 \% \mathrm{v} / \mathrm{v})$ and stained with crystal violet $(5 \% \mathrm{w} / \mathrm{v})$ for plaque

283 visualisation. The origin and characterization of the clinical isolate RSV BT2a were 284 previously described ${ }^{38}$. RSV titres in biological samples were determined using HEp-2 285 cells, as previously described ${ }^{39}$.

Well-differentiated primary nasal epithelial cell (WD-PNEC) culture, infection and 287 treatment

288 Nasal epithelial cells were obtained from consented healthy adults with no known history 289 of lung disease or previous SARS-CoV-2 infection. Culture and differentiation protocols 290 were previously described ${ }^{40,41}$. In brief, cells were passaged three times in Promocell 291 Airway Epithelial Cell Growth Medium (Promocell) then seeded onto collagen-coated 292 Transwell supports (Corning) at $3 \times 10^{4}$ cells per Transwell. After 4-6 days of submersion 293 air-liquid interface (ALI) was initiated by removing the apical medium. Cells were 294 differentiated using Stemcell PneumaCult ALI medium (Stemcell Technologies) 295 supplemented with hydrocortisone and heparin. Basolateral medium was replaced every 2962 days. Complete differentiation took a minimum of 21 days. WD-PNEC cultures were 297 only used when hallmarks of excellent differentiation were evident, including extensive 298 apical coverage with beating cilia and obvious mucus production. WD-PNECs were 299 infected with SARS-CoV-2 $(\mathrm{MOI}=0.1)$ by the addition of inoculum on the apical surface 
300 and incubation for $1 \mathrm{~h}$ at $37^{\circ} \mathrm{C}$. Cultures were treated basolaterally with $0,1 \mathrm{or} 100 \mathrm{ng} / \mathrm{mL}$

301 human IFN 33 (R\&D systems, Biotechne) for $24 \mathrm{~h}$. Ruxolitinib (Selleck Chemicals) $(1 \mu \mathrm{M})$

302 was added to the basolateral medium $48 \mathrm{~h}$ before infection and was replaced at 1 and 48

303 hpi. Apical washes were harvested by adding $200 \mu \mathrm{L}$ DMEM (low glucose; Gibco),

304 incubation for 5 mins and gentle aspiration without damaging the cultures.

\section{Immunofluorescence}

306 Cultures were fixed with $4 \%$ paraformaldehyde $(w / v)$ for a minimum of 1 hour. Cells were 307 permeabilised with $0.2 \%$ Triton $\mathrm{X}-100(\mathrm{v} / \mathrm{v})$ then blocked using $0.4 \%$ bovine serum 308 albumin (BSA) (w/v). Antibodies used are specified in figure legend. Cells were 309 counterstained with DAPI-mounting medium (Vectashield). Images were obtained on a 310 Nikon Eclipse TE-2000U or a Leica SP5 confocal microscope.

\section{Sequencing and Transcriptomic analysis}

312 WD-PNECs derived from 4 donors were infected with SARS-CoV-2 (MOI=0.1) or mock

313 infected. RNA was extracted at 48 and $96 \mathrm{~h}$ for each condition, in duplicate. Cultures

314 were incubated with $400 \mu \mathrm{l}$ of Lysis/Binding buffer (High Pure Isolation Kit; Roche) for 5

315 min at RT. Total Lysed samples were vortexed for $15 \mathrm{sec}$ and transferred to a High

316 Pure filter tube. RNA was extracted as per the manufacturer's instructions. RNA

317 sequencing was performed via Kapa RNA HyperPrep with RiboErase (Roche). Raw

318 sequence reads were quality checked with FastQC and pre-processed for the sequence

319 cleaning and adapter removal using cutadapt 2.1 and Trim Galore! To remove adapter

320 contamination. (https://www.bioinformatics.babraham.ac.uk/projects/trim galore/).

321 These pre-processed fastq files were then aligned to the human genome GRCh38v33

322 using STAR aligner version 2.7.3 $\mathrm{a}^{42}$ using default parameters and subsequently genes

323 were quantified using htseq version $0.11 .3^{43}$.

324 Quantified genes (raw counts) were processed using edge $\mathrm{R}^{44}$ for normalisation purposes 325 and transformed into logCPMs (log counts per million). These logCPM values were 326 subsequently used to generate a heatmap using pheatmap package for comparison and 327 visualisation purposes using a selection of interferon related genes extracted from a 
328 selection of pathways annotated in WikiPathways and Reactome Pathway Database

329 (WP2113; R-HSA-913531; WP1904; R-HSA-168928; WP4880; WP1835; R-HSA-

330 909733; WP75), ACE2 and TMPRSS2. SNP calling was carried out using ExactSNP

331 program from the subread package using default parameters ${ }^{45}$. To increase the

332 sequencing depth, we merged together all samples from each donor and used the

333 merged file as input for the SNP calling phase. In addition to SNP calling we also

334 annotated the variants identified using SnpEff software and the GRCh38.99 database ${ }^{46}$.

\section{ELISA analysis}

336 Concentrations of IFN- $\lambda 1$ in basolateral medium samples from WD-PNECs were 337 measured using a human IFN- $\lambda 1 /$ IL-29 ELISA (Invitrogen) according to the 338 manufacturer's instructions.

\section{Acknowledgements:}

342 The authors would like to thank the following individuals and groups for their contribution

343 and assistance: QUB FMHLS Genomics Core Technology Unit; QUB staff Grace Roberts, 344 Judit Barabas, Mervyn McCaigue, Cathy Fenning, Nuala McCann and David Norwood for 345 their guidance and assistance with biological safety and maintenance of the BSL3 346 laboratory.

\section{Funding:}

349 This study was supported by funding from UKRI/NIHR (MC_PC_19057) to UP and KM; 350 PHA HSCNI R\&D Division (COM/5613/20) to UP, KM, LB, CB, and GLC; and generous 351 donations from the public and QUB alumni to the Queen's University Belfast Foundation. 
354 1. Zhou, P. et al. A pneumonia outbreak associated with a new coronavirus of 355 probable bat origin. Nature 579, 270-273 (2020).

356 2. Zhu, N. et al. A Novel Coronavirus from Patients with Pneumonia in China, 2019. N. Engl. J. Med. 382, 727-733 (2020).

3. Xu, Z., Li, S., Tian, S., Li, H. \& Kong, L. quan. Full spectrum of COVID-19 severity still being depicted. The Lancet 395, 947-948 (2020).

4. Hamming, I. et al. Tissue distribution of ACE2 protein, the functional receptor for SARS coronavirus. A first step in understanding SARS pathogenesis. J. Pathol.

5. Shang, J. et al. Cell entry mechanisms of SARS-CoV-2. Proc. Natl. Acad. Sci. U. 203, 631-637 (2004).

6. Del Valle, D. M. et al. An inflammatory cytokine signature predicts COVID-19 severity and survival. Nat. Med. 26, 1636-1643 (2020).

7. Leisman, D. E. et al. Cytokine elevation in severe and critical COVID-19: a rapid systematic review, meta-analysis, and comparison with other inflammatory syndromes. The Lancet Respiratory Medicine 8, 1233-1244 (2020).

8. Shaw, A. E. et al. Fundamental properties of the mammalian innate immune system revealed by multispecies comparison of type I interferon responses. PLoS

9. Blanco-Melo, D. et al. Imbalanced Host Response to SARS-CoV-2 Drives Biol. 15, e2004086 (2017). 
11. Galani, I. E. et al. Untuned antiviral immunity in COVID-19 revealed by temporal type I/III interferon patterns and flu comparison. Nat. Immunol. 22, 32-40 (2021).

12. Sommereyns, C., Paul, S., Staeheli, P. \& Michiels, T. IFN-lambda (IFN-lambda) is expressed in a tissue-dependent fashion and primarily acts on epithelial cells in vivo. PLoS Pathog. 4, e1000017 (2008).

13. Jilg, N. et al. Kinetic differences in the induction of interferon stimulated genes by interferon- $\alpha$ and interleukin $28 \mathrm{~B}$ are altered by infection with hepatitis $\mathrm{C}$ virus. Hepatology 59, 1250-1261 (2014).

14. Zhou, S. et al. A Neanderthal OAS1 isoform protects individuals of European ancestry against COVID-19 susceptibility and severity. Nat. Med. 27, 659-667 (2021).

15. Pfaender, S. et al. LY6E impairs coronavirus fusion and confers immune control of viral disease. Nat. Microbiol. 5, 1330-1339 (2020).

16. Winstone, H. et al. The Polybasic Cleavage Site in SARS-CoV-2 Spike Modulates Viral Sensitivity to Type I Interferon and IFITM2. J. Virol. 95, (2021).

17. Vanderheiden, A. et al. Type I and Type III Interferons Restrict SARS-CoV-2 Infection of Human Airway Epithelial Cultures. J. Virol. 94, (2020).

18. Villenave, R. et al. In vitro modeling of respiratory syncytial virus infection of pediatric bronchial epithelium, the primary target of infection in vivo. Proc. Natl. Acad. Sci. U. S. A. 109, 5040-5 (2012).

19. Felgenhauer, U. et al. Inhibition of SARS-CoV-2 by type I and type III interferons. J. Biol. Chem. 295, 13958-13964 (2020).

20. Sungnak, W. et al. SARS-CoV-2 entry factors are highly expressed in nasal epithelial cells together with innate immune genes. Nat. Med. 2020265 26, 681687 (2020). 
402

21. Madewell, Z. J., Yang, Y., Longini, I. M., Halloran, M. E. \& Dean, N. E. Household Transmission of SARS-CoV-2: A Systematic Review and Meta-analysis. JAMA Netw. open 3, e2031756 (2020).

22. Grifoni, A. et al. Targets of T Cell Responses to SARS-CoV-2 Coronavirus in Humans with COVID-19 Disease and Unexposed Individuals. Cell 181, 14891501.e15 (2020).

23. Wickenhagen, A. et al. Wickenhagen et al., A Prenylated dsRNA Sensor Protects Against Severe COVID-19 and is Absent in Horseshoe Bats $1 \mathrm{~A}$ Prenylated dsRNA Sensor Protects Against Severe COVID-19 and is Absent in Horseshoe Bats. medRxiv 2021.05.05.21256681 (2021). doi:10.1101/2021.05.05.21256681

24. Dee, K. et al. Human rhinovirus infection blocks SARS-CoV-2 replication within the respiratory epithelium: implications for COVID-19 epidemiology. J. Infect. Dis. (2021). doi:10.1093/infdis/jiab147

25. Marcos-Villar, L. et al. Epigenetic control of influenza virus: Role of H3K79 methylation in interferon-induced antiviral response. Sci. Rep. 8, 1230 (2018).

26. Skevaki, C., Pararas, M., Kostelidou, K., Tsakris, A. \& Routsias, J. G. Single nucleotide polymorphisms of Toll-like receptors and susceptibility to infectious diseases. Clin. Exp. Immunol. 180, 165 (2015).

27. Huik, K. et al. Association between TLR3 rs3775291 and resistance to HIV among highly exposed Caucasian intravenous drug users. Infect. Genet. Evol. 20, 78 (2013).

28. $\mathrm{M}, \mathrm{S}$. et al. A common polymorphism in TLR3 confers natural resistance to HIV-1 infection. J. Immunol. 188, 818-823 (2012).

29. C, G. et al. A role for Toll-like receptor 3 variants in host susceptibility to enteroviral myocarditis and dilated cardiomyopathy. J. Biol. Chem. 285, 2320823223 (2010). 
428 30. Stockman, L. J., Bellamy, R. \& Garner, P. SARS: Systematic review of treatment effects. PLoS Medicine 3, 1525-1531 (2006).

31. Omrani, A. S. et al. Ribavirin and interferon alfa-2a for severe Middle East respiratory syndrome coronavirus infection: A retrospective cohort study. Lancet Infect. Dis. 14, 1090-1095 (2014).

32. Lazear, H. M., Schoggins, J. W. \& Diamond, M. S. Shared and Distinct Functions of Type I and Type III Interferons. Immunity 50, 907-923 (2019).

33. Pervolaraki, K. et al. Differential induction of interferon stimulated genes between type I and type III interferons is independent of interferon receptor abundance. PLoS Pathog. 14, e1007420 (2018).

34. Donnelly, R. P., Dickensheets, H. \& O’Brien, T. R. Interferon-lambda and therapy for chronic hepatitis C virus infection. Trends Immunol. 32, 443-50 (2011).

35. Giersch, K. et al. Both interferon alpha and lambda can reduce all intrahepatic HDV infection markers in HBV/HDV infected humanized mice. Sci. Reports 2017

36. Feld, J. J. et al. Peginterferon lambda for the treatment of outpatients with $717,1-11(2017)$.

37. Bamford, C. G. G. et al. Collaboration Between Host and Viral Factors Shape SARS-CoV-2 Evolution. bioRxiv 2021.07.16.452629 (2021).

38. Villenave, R. et al. Differential cytopathogenesis of respiratory syncytial virus prototypic and clinical isolates in primary pediatric bronchial epithelial cells. Virol. J. 8, 43 (2011).

39. Power, U. F. et al. Induction of Protective Immunity in Rodents by Vaccination 
Respiratory Syncytial Virus G Protein Fragment. Virology 230, 155-166 (1997).

455

40. Broadbent, L. et al. In Vitro Modeling of RSV Infection and Cytopathogenesis in Well-Differentiated Human Primary Airway Epithelial Cells (WD-PAECs). in Methods in molecular biology (Clifton, N.J.) 1442, 119-39 (2016).

41. Broadbent, L. et al. Comparative primary paediatric nasal epithelial cell culture differentiation and RSV-induced cytopathogenesis following culture in two commercial media. PLoS One 15, (2020).

42. Dobin, A. et al. STAR: Ultrafast universal RNA-seq aligner. Bioinformatics 29, 15$21(2013)$.

43. Anders, S., Pyl, P. T. \& Huber, W. HTSeq-A Python framework to work with highthroughput sequencing data. Bioinformatics 31, 166-169 (2015).

44. Robinson, M. D., McCarthy, D. J. \& Smyth, G. K. edgeR: A Bioconductor package for differential expression analysis of digital gene expression data. Bioinformatics 26, 139-140 (2009).

45. Y, L., GK, S. \& W, S. The R package Rsubread is easier, faster, cheaper and better for alignment and quantification of RNA sequencing reads. Nucleic Acids Res. 47, (2019).

46. Cingolani, P. et al. A program for annotating and predicting the effects of single nucleotide polymorphisms, SnpEff: SNPs in the genome of Drosophila melanogaster strain w1118; iso-2; iso-3. Fly (Austin). 6, 80 (2012). 
A

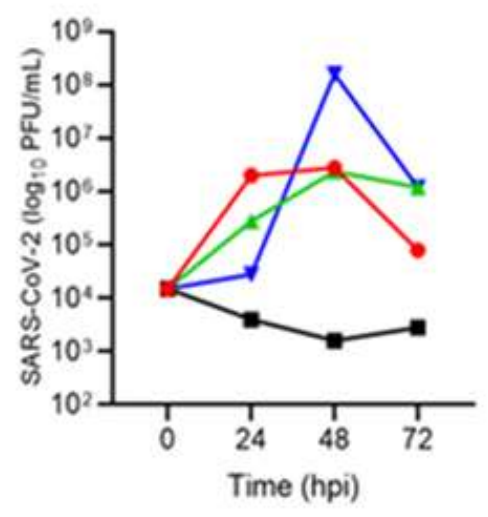

C

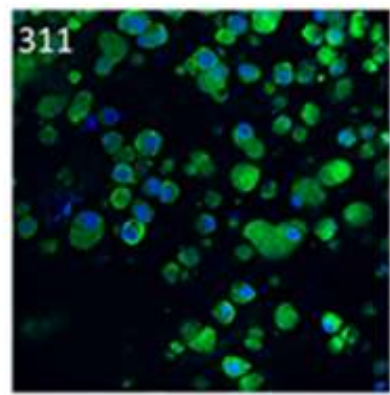

479

480

481

482

483

484

485

486

487

488

489

490

\section{2}
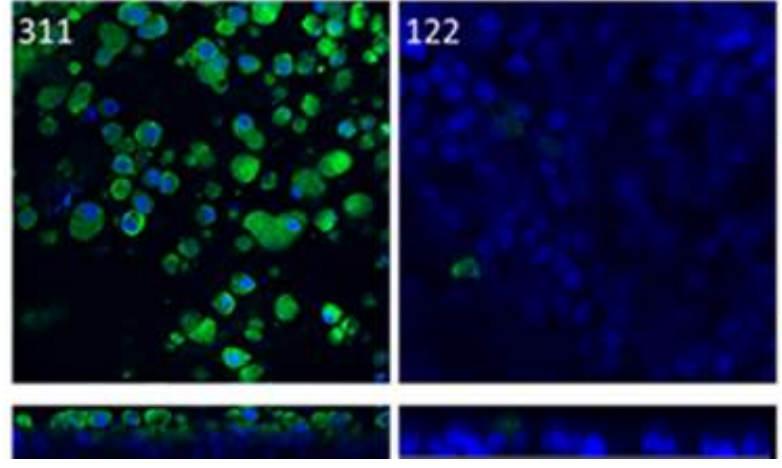

- 311

$-122$

- 43

- 32

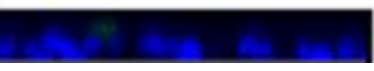

B
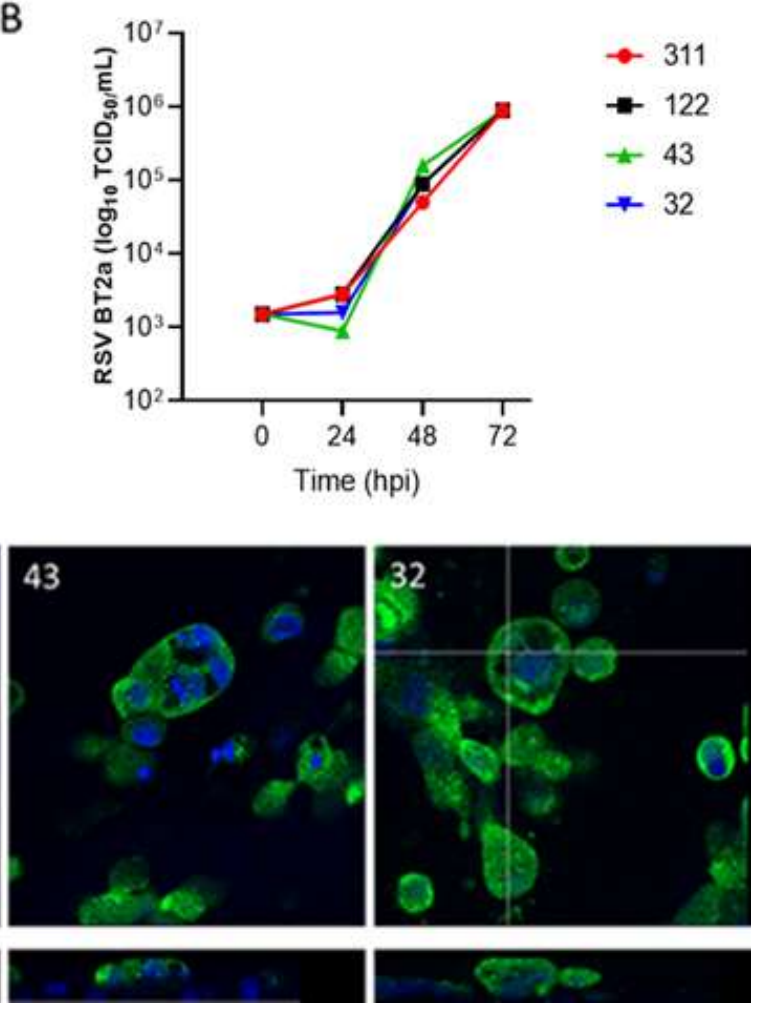
bioRxiv preprint doi: https://doi.org/101101/2021.08.17.456707- this version posted August 18,2021 . The copyright holder for this preprint (which was not certified by peer review) is the author/funder, who has granted bioRxiv a license to display the preprint in perpetuity. It is made available under aCC-BY-NC-ND 4.0 International license.

A

494

Heatmap of Selected IFN genes (scaled)

495
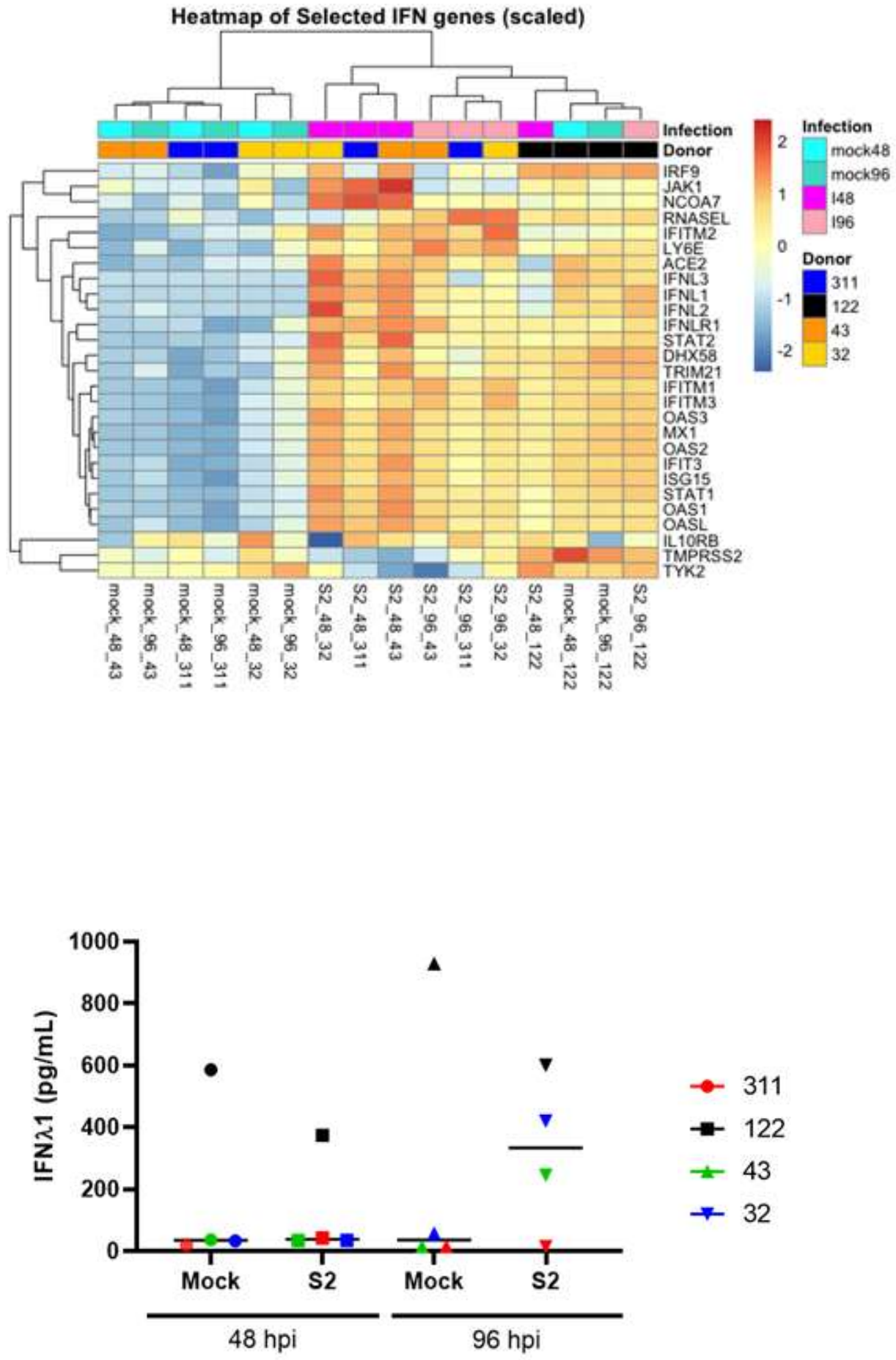
512 Figure 2. Transcriptomic profiles and protein quantification demonstrated a 513 preactivated IFN response in a donor resistant to infection.

514 WD-PNECs from 4 adult donors were infected with a clinical isolate of SARS-CoV-2

515 (MOl=0.1) or mock infected. At 48 and 96 hpi RNA was extracted from the cultures and 516 global transcriptomic analysis was performed. Selected genes, pertaining to innate 517 immune responses to viral infection are illustrated by heatmap, gene upregulation (Red) 518 and down regulation (blue) (A). Preactivated immune response was validated by 519 quantifying IFN $\lambda 1$ concentration in basolateral medium from mock and SARS-CoV-2 520 infected WD-PNECs from 4 donors was quantified by ELISA (B). 
A

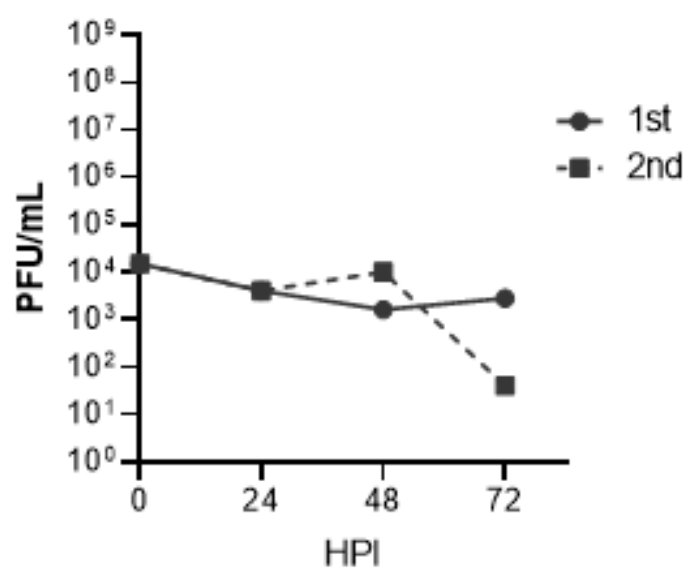

B

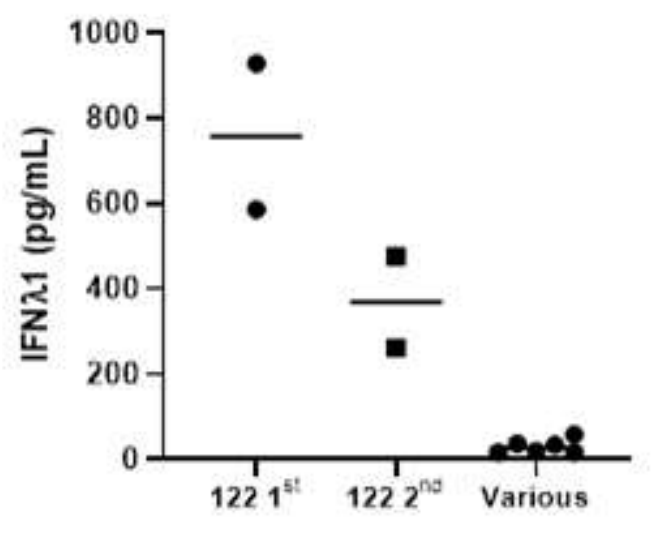
was not transient

540 Independent nasal brushes were obtained from donor 122 four months apart, designated

$5411^{\text {st }}$ brush and $2^{\text {nd }}$ brush ( $1^{\text {st }}$ brush results from experiment $1 \mathrm{~A}$ ). Cells were cultured for a 542 minimum of 21 days in ALI until fully differentiated then infected with SARS-CoV-2 543 (MOI=0.1). Apical washes were harvested every $24 \mathrm{hpi}$ up to $72 \mathrm{hpi}$ and titrated by plaque 544 assay. (A). IFN $\lambda$ concentration in basaolateral medium from uninfected WD-PNECs from 545 the $1^{\text {st }}$ and $2^{\text {nd }}$ nasal brushes from donor 122 were determined by ELISA. This is 546 compared to other uninfected WD-PNEC cultures derived from 6 donors (B). 
A

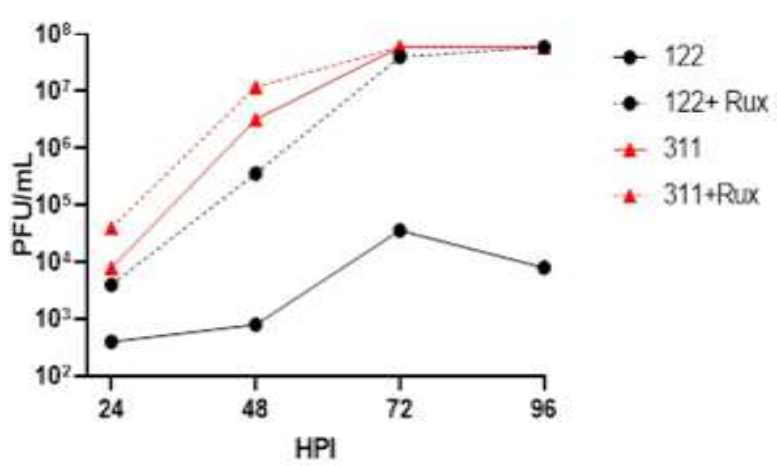

B

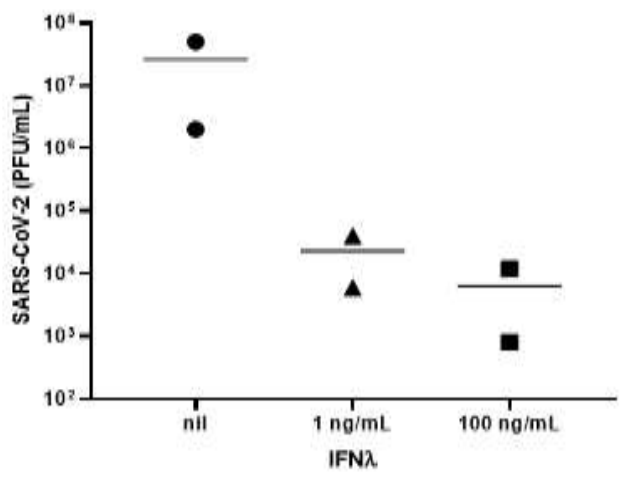
Figure 4. SARS-CoV-2 growth kinetics are dependent on IFN signalling. WD-PNECs

558 derived from donor 122 and a permissive donor were treated basolaterally with $1 \mu \mathrm{M}$ 559 ruxolitinib for $48 \mathrm{~h}$, or left untreated, prior to infection with SARS-CoV-2 (MOI=0.1).

560 Ruxolitinib was replaced at 1 and $48 \mathrm{hpi}$. Apical washes were harvested every $24 \mathrm{~h}$ post 561 infection until 96 hpi. SARS-CoV-2 viral growth kinetics were determined by plaque assay 562 on Vero cells (A). WD-PNECs derived from a SARS-CoV-2 permissive donor were treated 563 basolaterally in duplicate with $0,1 \mathrm{ng}$ or $100 \mathrm{ng} / \mathrm{mL}$ IFN $\lambda$ for $24 \mathrm{~h}$ prior to infection with 564 SARS-CoV-2 $(\mathrm{MOI}=0.1)$. Apical washes were harvested at $48 \mathrm{hpi}$ and titrated by plaque 565 assay on Vero cells. 


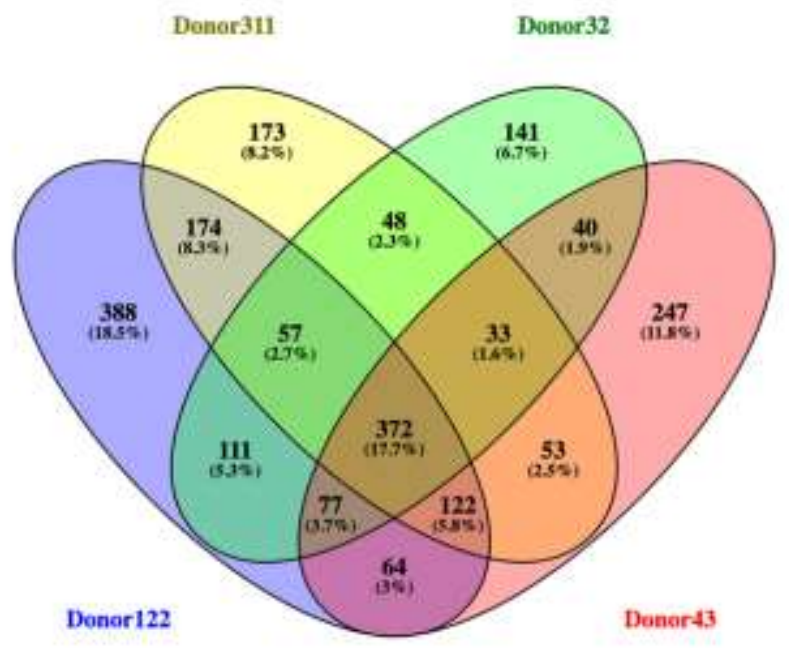

\begin{tabular}{|c|l|c|}
\hline \multicolumn{1}{|c|}{ SNP } & \multicolumn{1}{|c|}{ Gene } & Donor \\
\hline rs3738032 & ADAR & 122 \\
\hline rs1127313 & ADAR & 311 \\
& & 43 \\
& & 32 \\
\hline rs1127326 & ADAR & 311 \\
& & 43 \\
& & 32 \\
\hline rs1990760 & IFIH1/MDA-5 & 311 \\
& & 43 \\
& & 122 \\
\hline rs2089960995 & MAVS & 122 \\
\hline rs4833095 & TLR1 & 122 \\
\hline rs1816702 & TLR2 & 122 \\
\hline rs3804099 & TLR2 & 122 \\
\hline rs3775296 & TLR3 & \\
\hline rs3775291 & TLR3 & \\
\hline & & \\
\hline
\end{tabular}

574 donor 122 were filtered by quality $(>10)$, with an existing entry in dbSNP $(\mathrm{NIH})$ and previously published (B). 